\title{
Equilibrium Under Moral Hazard
}

\author{
by David Gale *
}

The breakdown of the results of classical equilibrium theory in the presence of moral hazard or, what really matters, unobservable actions, is a currently popular and timely subject. Professor Stiglitz's paper studies this phenomenon in the context of insurance contracts but, of course, the phenomenon is much more general and is essentially quite independent of any consideration of risk or even uncertainty. For example, as I was about to leave my hotel room this morning I realized that the light was still on in the bathroom. For me there was no incentive to reenter the room and turn out the light since my hotel bill would not be affected. As a rational utility maximizer I therefore locked the door and proceeded on my way. (Whether or not this act was "moral" is beyond the scope of the present discussion.) Now assuming, as in the Stiglitz model that all consumers are identical, i.e. just like me, hotels in order to insure zero profit are required to take account of our laziness in setting their rates for rooms. This in general leads to a welfare loss for us consumers since if the hotels could be sure we would always turn out lights it could probably make a more than compensating reduction in our bill.

In this discussion I will concentrate on the first part of Stiglitz's paper and try to illustrate in another way how quantity rationing may solve the equilibrium problem when transactions are observable. I will use the Stiglitz scenario of the home owner who must make two decisions : (1) whether or not to smoke and (2) whether or not to insure. In Stiglitz's first model the home owner has no control over the extent of his insurance coverage but must either accept or reject the policy which the company offers.

In the formal model let $p$ be the probability of a fire in case the home owner does not smoke. Then in order to insure zero profits the insurance company must choose premium $\beta$ and benefit $\alpha$ so that $\beta / \alpha=p /(1-p)$. In the Stiglitz model the owner's satisfaction depends only on his expected income, so let us normalize units so that his income is 0 if there is a fire and 1 if not. Similarly we may normalize utility so that $u(0)=0$ and $u(1)=1$. Then with no insurance the home owner's expected utility will be :

$$
p u(0)+(1-p) u(1)=1-p
$$

* University of California, Berkeley. 
Now if we introduce insurance with premium $\lambda p$ and benefit $\lambda(1-p)$ where $0 \leqslant \lambda \leqslant 1$ we have expected utility

$$
p u(\lambda(1-p))+(1-p) u(1-\lambda p)
$$

and by concavity of $u$ this is preferred to no insurance given by (1). In fact one easily verifies by differentiating (2) that the owner would like full coverage, that is $\lambda=1$.

Now we introduce the possibility of smoking. Under the rather natural assumption that the pleasure of smoking is independent of the amount of income, we simply add a constant $e$ to the utility function. However smoking also increases the probability of fire from $p$ to some value $p^{\prime}$. The first question to be settled is whether in the absence of insurance the owner would smoke or not. For the analysis of moral hazard the interesting case is when he would not, i.e. exactly when $1-p^{\prime}+e<1-p$ or

$$
p+e<p^{\prime} \text {. }
$$

Now imagine insurance companies appearing on the scene. They will then be able to sell insurance with premium and benefit $(\lambda p,(1-\lambda) p)$ but they dare not provide full coverage $(\lambda=1)$ because in that case the insured will certainly smoke, since he receives the same income, fire or not, so there will be more fires and the companies will be put out of business. Thus the companies must choose $\lambda$ up to but not beyond the point where the owners will smoke. Let us denote this value of $\lambda$ by $\bar{\lambda}$.

Now there is a second possibility. The insurance companies may provide more expensive insurance with premium and benefit $\left(\lambda p^{\prime},(1-\lambda) p^{\prime}\right)$, under the assumption that the owners will definitely smoke. In this case the companies will be able to offer full coverage and the guarantied utility of the owners will be $u\left(1-p^{\prime}\right)$. Now the question is, which will be the equilibrium policy, the expensive one with full coverage or the cheaper one with partial coverage. The answer is that it will be the one the owners prefer. More precisely it will be the expensive policy if and only if

$$
u\left(1-p^{\prime}\right) \geqq p u(\bar{\lambda} p)+(1-p) u(1-\bar{\lambda} p)
$$

Since the inequality above can go in either direction one does not know in advance whether the cheap or expensive insurance will give equilibrium. The more interesting case is the one in which the cheaper insurance gives equilibrium. If one thinks of equilibrium as being reached through competition among insurance companies then the owners have the choice between full coverage at a high premium or partial coverage at a low one. The natural extension of this phenomenon would be a non-linear premium which increased according to the amount of coverage. It would be interesting to see to what extent such phenomena are observed in actual insurance markets. 